\title{
Statistical Modeling of a Ligand Knowledge Base
}

\author{
Ralph A. Mansson, Alan H. Welsh, Natalie Fey and A. Guy Orpen
}

Supporting Information 
Table S1: Values of Tolman's Electronic Parameter, $\mathrm{A}_{1} v(\mathrm{CO})$ in $\left[(\mathrm{CO})_{3} \mathrm{NiL}\right]$, for LKB; Ligand Weightings in Robust Models

\begin{tabular}{|c|c|c|c|c|c|}
\hline No. & Ligand & TEP $\left(\mathrm{cm}^{-1}\right)$ & $\begin{array}{c}\text { From substituent } \\
\text { contributions? }\end{array}$ & $\begin{array}{c}\text { Weights in } \\
\text { RLM1 }\end{array}$ & $\begin{array}{c}\text { Weights in } \\
\text { RLM2 }\end{array}$ \\
\hline 1 & $\mathrm{PH}_{3}$ & 2083.2 & & 0.9992 & 0.9954 \\
\hline 2 & $\mathrm{PMe}_{3}$ & 2064.1 & & 0.9966 & 0.9889 \\
\hline 3 & $\mathrm{PEt}_{3}$ & 2061.7 & & 0.9839 & 0.9975 \\
\hline 4 & $\mathrm{PPr}_{3}$ & & & & \\
\hline 5 & $\mathrm{P}^{\mathrm{i}} \mathrm{Pr}_{3}$ & 2059.2 & & 0.9947 & 0.9887 \\
\hline 6 & $\mathrm{PBu}_{3}$ & 2060.3 & & 0.9892 & 0.9545 \\
\hline 7 & $\mathrm{P}^{\mathrm{t}} \mathrm{Bu}_{3}$ & 2056.1 & & 0.9463 & 0.9926 \\
\hline 8 & $\mathrm{P}\left(\mathrm{CF}_{3}\right)_{3}$ & 2114.9 & $\checkmark$ & 0 & 0.9976 \\
\hline 9 & $\mathrm{PCy}_{3}$ & 2056.4 & & 0.9638 & 0.8922 \\
\hline 10 & $\mathrm{PBz}_{3}$ & 2066.4 & & 0 & 0.9727 \\
\hline 11 & $\mathrm{PF}_{3}$ & 2110.8 & & 0.9863 & 0.9753 \\
\hline 12 & $\mathrm{PCl}_{3}$ & 2097.0 & & 0.8572 & 0.8998 \\
\hline 13 & $\mathrm{P}(\mathrm{OMe})_{3}$ & 2079.5 & & 0.9639 & 0.8957 \\
\hline 14 & $\mathrm{P}(\mathrm{OEt})_{3}$ & 2076.3 & & 0.9994 & 0.6348 \\
\hline 15 & $\mathrm{P}(\mathrm{OPh})_{3}$ & 2085.3 & & 0.8860 & 0.9494 \\
\hline 16 & $\mathrm{P}\left(\mathrm{NH}_{2}\right)_{3}$ & & & & \\
\hline 17 & $\mathrm{P}\left(\mathrm{NMe}_{2}\right)_{3}$ & 2061.9 & & 0.8780 & 0 \\
\hline 18 & $\mathrm{PPyr}_{3}$ & & & & \\
\hline 19 & $\mathrm{P}\left(\mathrm{NC}_{4} \mathrm{H}_{8}\right)_{3}$ & & & & \\
\hline 20 & $\mathrm{PPip}_{3}$ & 2062.1 & $\checkmark$ & 0.9884 & 0 \\
\hline 21 & $\mathrm{P}\left(\mathrm{CHCH}_{2}\right)_{3}$ & 2069.5 & & 0.9998 & 0.9536 \\
\hline 22 & $\mathrm{PPh}_{3}$ & 2068.9 & & 0.9223 & 0.9297 \\
\hline 23 & $\mathrm{P}\left(\mathrm{C}_{6} \mathrm{~F}_{5}\right)_{3}$ & 2090.9 & & 0.8792 & 0.9310 \\
\hline 24 & $\mathrm{P}(\mathrm{o}-\mathrm{Me}-\mathrm{Ph})_{3}$ & 2066.6 & & 0.9969 & 0.9761 \\
\hline 25 & $\mathrm{P}(\mathrm{m}-\mathrm{Me}-\mathrm{Ph})_{3}$ & 2067.2 & & 0.9903 & 0.9790 \\
\hline 26 & $\mathrm{P}(\mathrm{p}-\mathrm{Me}-\mathrm{Ph})_{3}$ & 2066.7 & & 0.9414 & 0.6049 \\
\hline 27 & $\mathrm{P}(\mathrm{o}-\mathrm{MeO}-\mathrm{Ph})_{3}$ & 2058.3 & & 0.9839 & 0.9577 \\
\hline 28 & $\mathrm{P}(\mathrm{p}-\mathrm{MeO}-\mathrm{Ph})_{3}$ & 2066.1 & & 0.8070 & 0.2423 \\
\hline 29 & $\mathrm{P}\left(3,5-\left(\mathrm{CF}_{3}\right)_{2}-\mathrm{Ph}\right)_{3}$ & & & & \\
\hline 30 & $\mathrm{P}\left(\mathrm{p}-\mathrm{F}_{3} \mathrm{C}-\mathrm{Ph}\right)_{3}$ & & & & \\
\hline 31 & $\mathrm{P}(\mathrm{p}-\mathrm{F}-\mathrm{Ph})_{3}$ & 2071.3 & & & 0.9559 \\
\hline 32 & $\mathrm{P}(\mathrm{p}-\mathrm{Cl}-\mathrm{Ph})_{3}$ & 2072.8 & & & 0.9954 \\
\hline 33 & $\mathrm{P}\left(\mathrm{p}-\mathrm{Me}_{2} \mathrm{~N}-\mathrm{Ph}\right)_{3}$ & & & & \\
\hline 34 & $\mathrm{PHF}_{2}$ & 2100.8 & $\checkmark$ & 0.6095 & 0.9341 \\
\hline 35 & $\mathrm{PFH}_{2}$ & 2090.9 & $\checkmark$ & 0.9640 & 0.9132 \\
\hline 36 & $\mathrm{PHCl}_{2}$ & 2094.0 & $\checkmark$ & 0.9186 & 0.9333 \\
\hline 37 & $\mathrm{PClH}_{2}$ & 2087.5 & $\checkmark$ & 0.9575 & 0.9685 \\
\hline 38 & $\mathrm{PMeF}_{2}$ & 2095.1 & $\checkmark$ & 0.8246 & 0.7916 \\
\hline 39 & $\mathrm{PFMe}_{2}$ & 2079.5 & $\checkmark$ & 0.7160 & 0.9988 \\
\hline 40 & $\mathrm{PMeCl}_{2}$ & 2088.3 & $\checkmark$ & 0.9183 & 0.7530 \\
\hline 41 & $\mathrm{PClMe}_{2}$ & 2076.1 & $\checkmark$ & 0.9504 & 0.9969 \\
\hline 42 & $\mathrm{PMe}\left(\mathrm{CF}_{3}\right)_{2}$ & 2097.9 & $\checkmark$ & 0 & 0.6639 \\
\hline 43 & $\mathrm{P}\left(\mathrm{CF}_{3}\right) \mathrm{Me}_{2}$ & 2080.9 & & 0.6743 & 0.8758 \\
\hline
\end{tabular}


Table S1 (Cont'd)

\begin{tabular}{|c|c|c|c|c|c|}
\hline No. & Ligand & TEP $\left(\mathrm{cm}^{-1}\right)$ & $\begin{array}{c}\text { From substituent } \\
\text { contributions? }\end{array}$ & $\begin{array}{l}\text { Weights in } \\
\text { RLM1 }\end{array}$ & $\begin{array}{l}\text { Weights in } \\
\text { RLM2 }\end{array}$ \\
\hline 44 & $\mathrm{PMe}^{\mathrm{t}} \mathrm{Bu}_{2}$ & 2058.7 & $\checkmark$ & 0.9999 & 0.8634 \\
\hline 45 & $\mathrm{P}^{t} \mathrm{BuMe}_{2}$ & 2061.3 & $\checkmark$ & 0.9821 & 0.9806 \\
\hline 46 & $\mathrm{PMePh}_{2}$ & 2067.0 & & 0.9596 & 0 \\
\hline 47 & $\mathrm{PPhMe}_{2}$ & 2065.3 & & 0.9708 & 0.9772 \\
\hline 48 & $\mathrm{PEtPh}_{2}$ & 2066.7 & & 0.8646 & 0.9607 \\
\hline 49 & $\mathrm{PPhEt}_{2}$ & 2063.7 & & 0.9974 & 0.9963 \\
\hline 50 & $\mathrm{PCyPh}_{2}$ & 2064.8 & $\checkmark$ & 0.8455 & 0.9885 \\
\hline 51 & $\mathrm{PPhCy}_{2}$ & 2060.5 & $\checkmark$ & 0.9742 & 0.7346 \\
\hline 52 & $\mathrm{PPyrPh}_{2}$ & & & & \\
\hline 53 & $\mathrm{PPhPyr}_{2}$ & & & & \\
\hline 54 & $\mathrm{P}(\mathrm{o}-\mathrm{Me}-\mathrm{Ph}) \mathrm{Ph}_{2}$ & 2068.2 & $\checkmark$ & 0.9958 & 0.9840 \\
\hline 55 & $\mathrm{PPh}(\mathrm{o}-\mathrm{Me}-\mathrm{Ph})_{2}$ & 2067.4 & $\checkmark$ & 0.9975 & 0.9990 \\
\hline 56 & $\mathrm{P}(\mathrm{o}-\mathrm{MeO}-\mathrm{Ph}) \mathrm{Ph}_{2}$ & 2066.1 & & 0.9910 & 0.9959 \\
\hline 57 & $\mathrm{PPh}(\mathrm{o}-\mathrm{MeO}-\mathrm{Ph})_{2}$ & 2062.2 & $\checkmark$ & 0.9870 & 0.9981 \\
\hline \multirow[t]{2}{*}{58} & & & & 0.7472 & 0.9979 \\
\hline & & 2087.3 & & & \\
\hline \multicolumn{6}{|l|}{59} \\
\hline \multicolumn{6}{|l|}{60} \\
\hline & 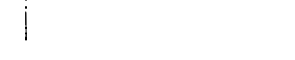 & & & & \\
\hline 61 & & & & & \\
\hline
\end{tabular}


Table S2: Descriptor Coefficients for TEP Models (OLS, RLM, LASSO, LAR)

\begin{tabular}{|c|c|}
\hline Descriptor & Coefficient \\
\hline \multicolumn{2}{|l|}{ Model: OLS } \\
\hline (Intercept) & 2555.28 \\
\hline PA & -0.52 \\
\hline LP s-character & -0.65 \\
\hline $\mathrm{Q}$ (Pt fragm.) & -47.79 \\
\hline$\Delta \mathrm{A}-\mathrm{P}-\mathrm{A}(\mathrm{Pd})$ & -2.18 \\
\hline P-B & 253.16 \\
\hline $\mathrm{P}-\mathrm{Pt}$ & -350.17 \\
\hline \multicolumn{2}{|l|}{ Model: RLM1 } \\
\hline (Intercept) & 3263.42 \\
\hline PA & -0.39 \\
\hline LP s-character & -0.64 \\
\hline $\mathrm{Q}(\mathrm{Pt}$ fragm. $)$ & 41.06 \\
\hline $\mathrm{BE}(\mathrm{Pd})$ & -0.24 \\
\hline$\Delta \mathrm{A}-\mathrm{P}-\mathrm{A}(\mathrm{Pd})$ & -1.73 \\
\hline $\mathrm{P}-\mathrm{Pd}$ & -68.74 \\
\hline $\mathrm{P}-\mathrm{Pt}$ & -174.22 \\
\hline $\mathrm{He}_{8 \_ \text {steric }}$ & 0.34 \\
\hline Pd-Cl trans & -209.44 \\
\hline \multicolumn{2}{|l|}{ Model: RLM2 } \\
\hline (Intercept) & 2605.61 \\
\hline $\mathrm{E}_{\mathrm{HOMO}}$ & -215.36 \\
\hline $\mathrm{Q}(\mathrm{Pt}$ fragm. $)$ & -9.98 \\
\hline$\Delta \mathrm{P}-\mathrm{A}(\mathrm{B})$ & 528.37 \\
\hline$\Delta \mathrm{A}-\mathrm{P}-\mathrm{A}(\mathrm{Pt})$ & -0.94 \\
\hline $\mathrm{P}-\mathrm{Pt}$ & -243.71 \\
\hline $\mathrm{He}_{8 \_ \text {steric }}$ & 0.16 \\
\hline \multicolumn{2}{|l|}{ Model: LASSO } \\
\hline $\mathrm{PA}$ & -6.33 \\
\hline $\mathrm{E}_{\mathrm{HOMO}}$ & -2.78 \\
\hline LP s-character & -2.73 \\
\hline $\mathrm{BE}(\mathrm{B})$ & -0.73 \\
\hline $\mathrm{BE}(\mathrm{Pd})$ & -0.05 \\
\hline$\Delta \mathrm{P}-\mathrm{A}(\mathrm{B})$ & 1.86 \\
\hline$\Delta \mathrm{P}-\mathrm{A}(\mathrm{Pt})$ & -1.26 \\
\hline$\Delta \mathrm{A}-\mathrm{P}-\mathrm{A}(\mathrm{B})$ & -0.25 \\
\hline$\Delta \mathrm{A}-\mathrm{P}-\mathrm{A}(\mathrm{Pd})$ & -1.72 \\
\hline$\Delta \mathrm{A}-\mathrm{P}-\mathrm{A}(\mathrm{Pt})$ & -0.17 \\
\hline P-B & 0.52 \\
\hline $\mathrm{P}-\mathrm{Pt}$ & -10.04 \\
\hline $\mathrm{He}_{8 \_ \text {steric }}$ & 1.78 \\
\hline$<\left(\mathrm{H}_{3} \mathrm{P}\right) \mathrm{Pt}\left(\mathrm{PH}_{3}\right)$ & -0.20 \\
\hline
\end{tabular}


Table S2: Cont'd

\begin{tabular}{|l|r|}
\hline Descriptor & Coefficient \\
\hline Model: $L A R$ & \\
\hline $\mathrm{PA}$ & -1.86 \\
\hline EHOMO & -4.95 \\
\hline$\Delta \mathrm{P}-\mathrm{A}(\mathrm{B})$ & 1.46 \\
\hline$\Delta \mathrm{A}-\mathrm{P}-\mathrm{A}(\mathrm{B})$ & -0.86 \\
\hline$\Delta \mathrm{A}-\mathrm{P}-\mathrm{A}(\mathrm{Pd})$ & -0.31 \\
\hline $\mathrm{P}-\mathrm{Pt}$ & -6.46 \\
\hline $\mathrm{Pd}-\mathrm{Cl}$ trans & -1.09 \\
\hline
\end{tabular}

Figure S1: Diagnostic Plot for OLS Model.

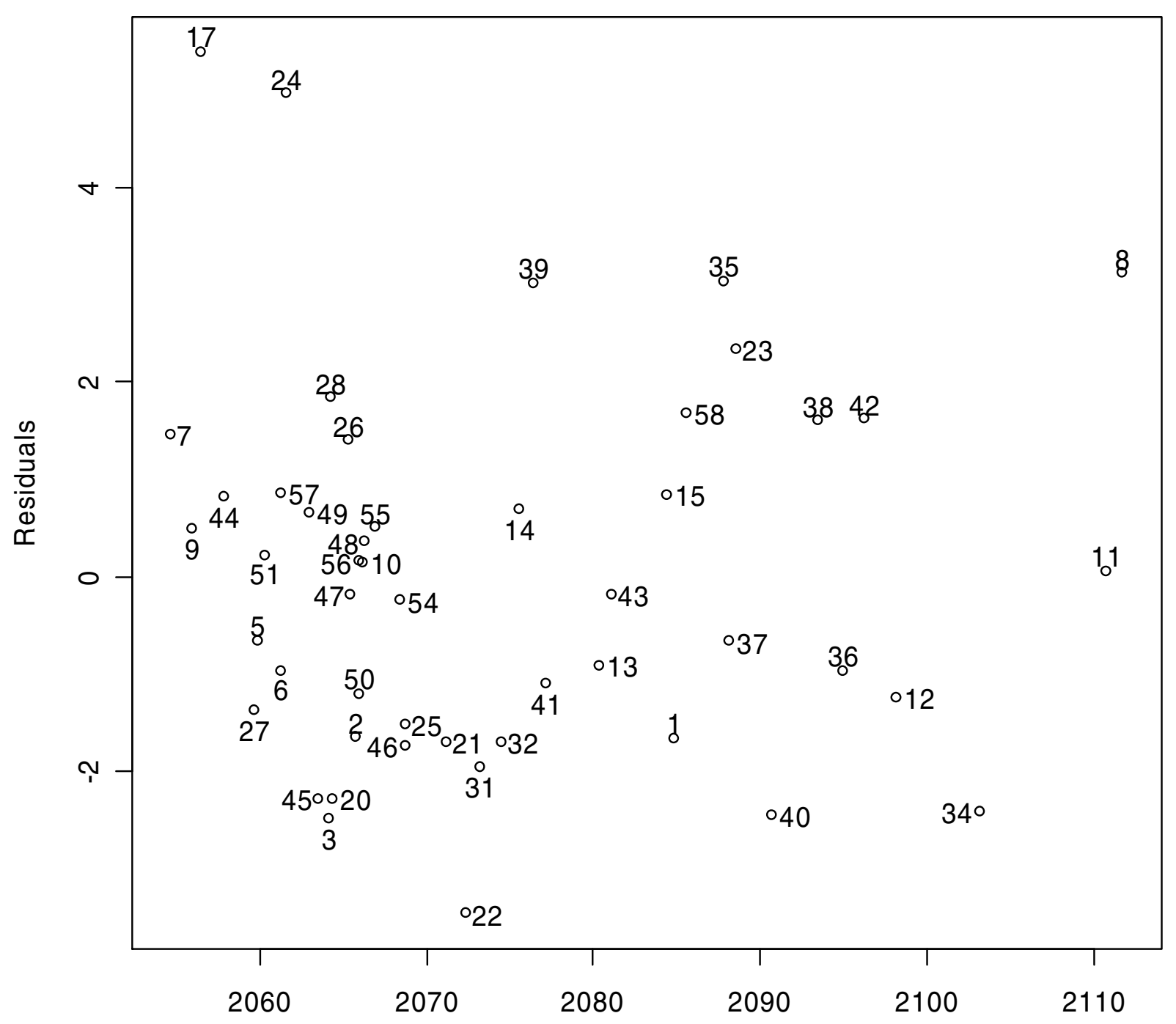

Fitted 
Figure S2: Diagnostic Plots for Robust Linear Regression Models (incl. indication of weightings), a) RLM1

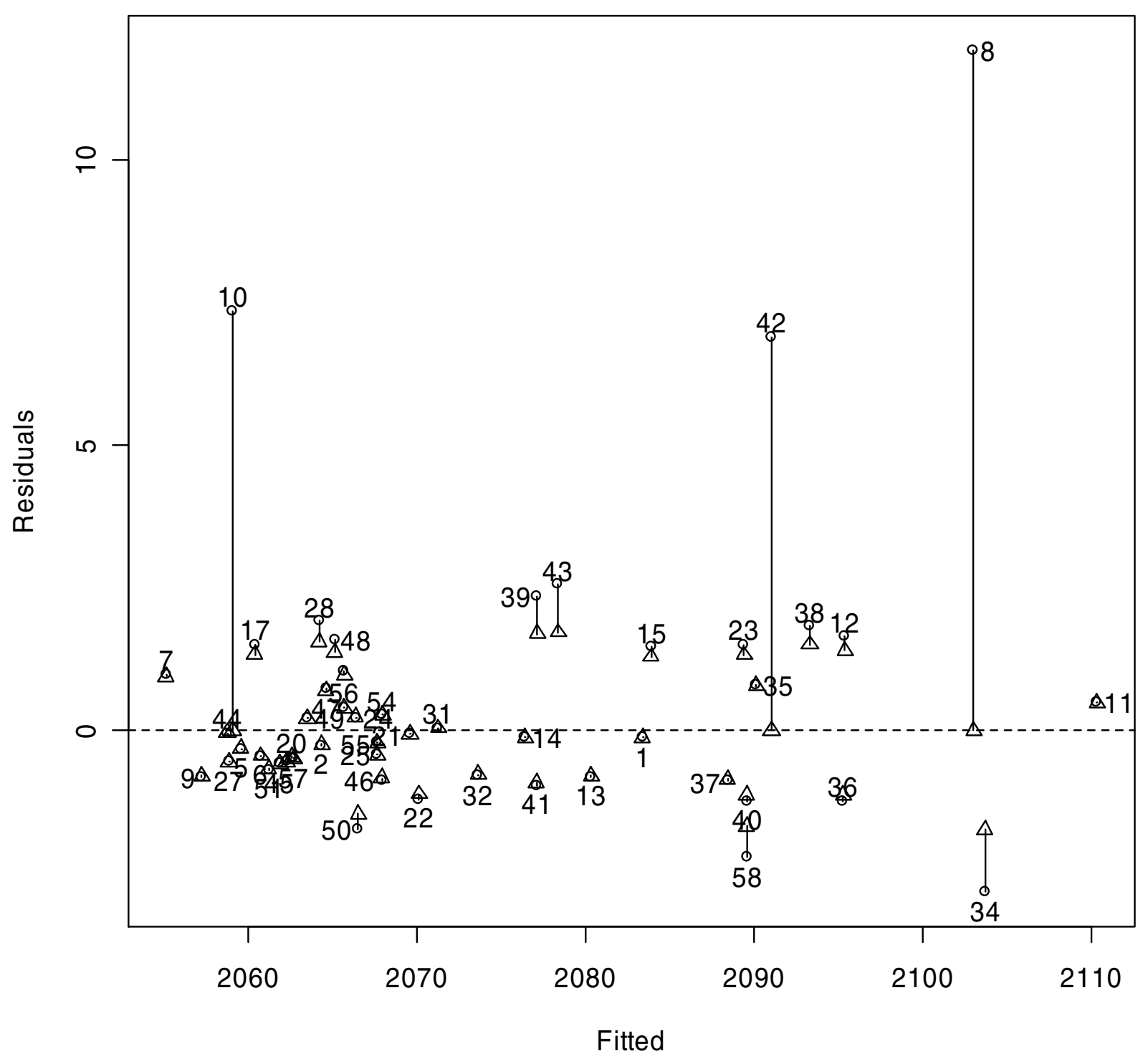


Figure S2: Cont'd, b) RLM2

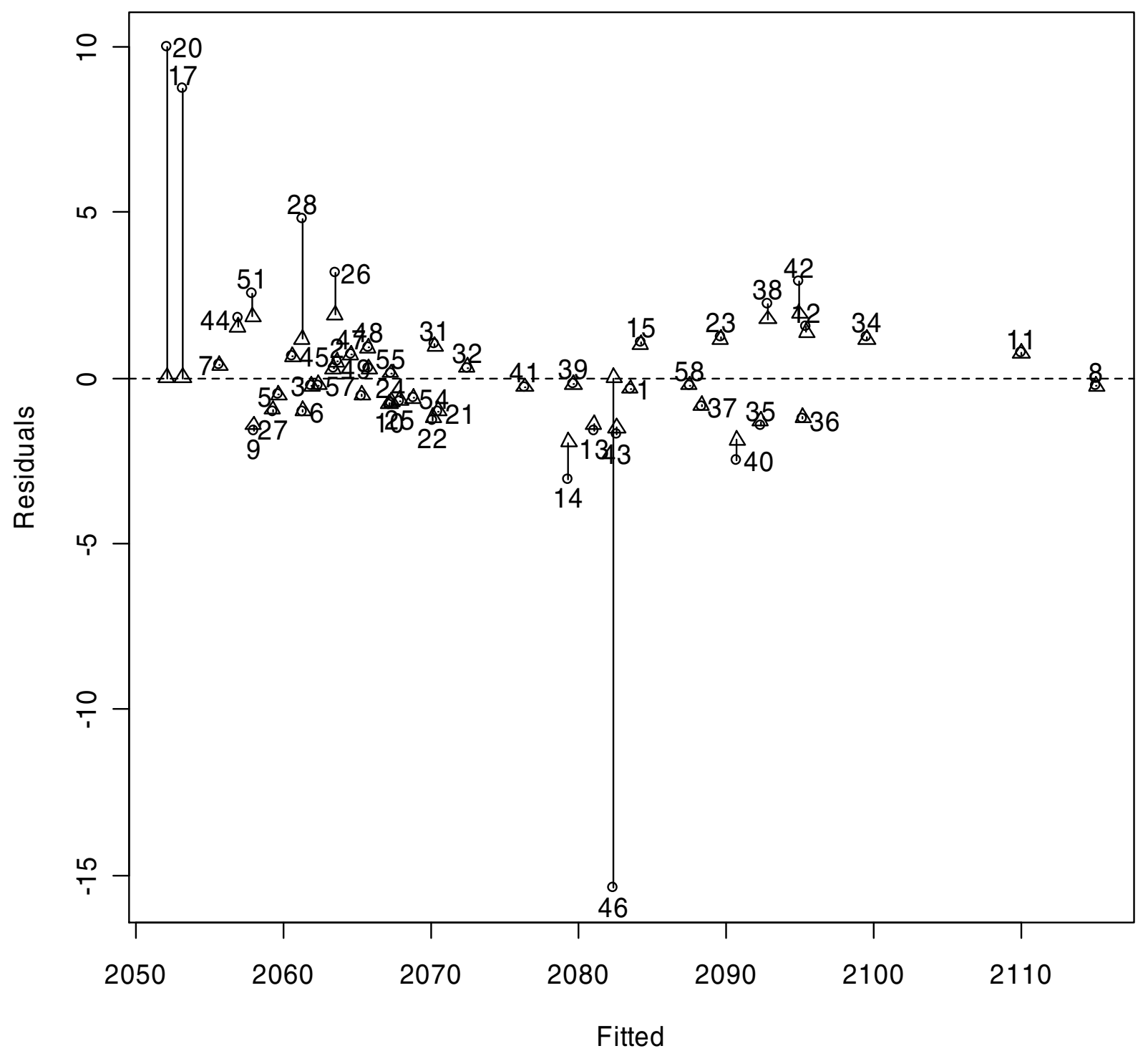


Figure S3: Diagnostic Plots for LASSO and LAR

a) LASSO, i) Plot of 10-fold Crossvalidation Values against Tuning Parameter

\section{LASSO}

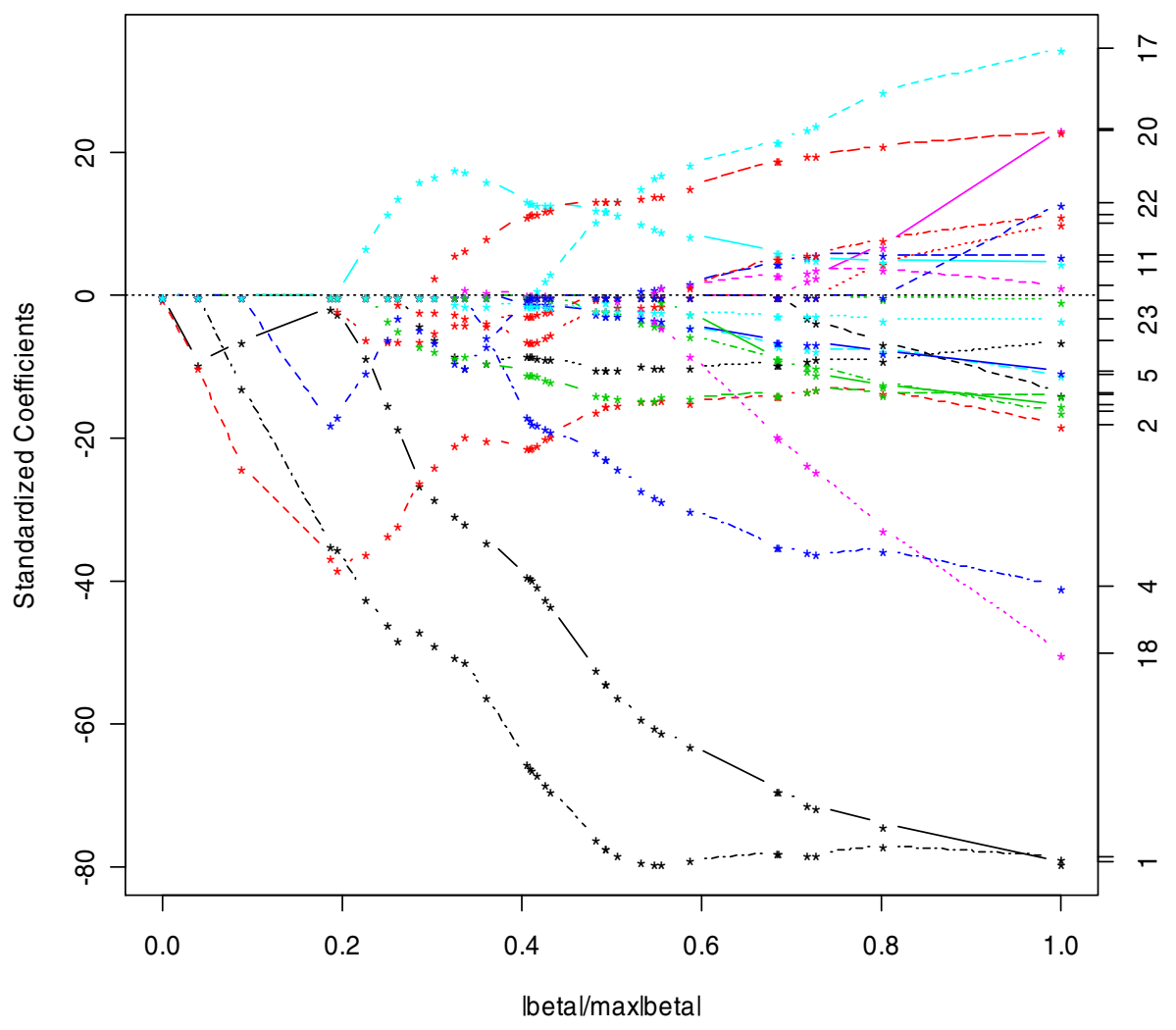

ii) Cross-validation Estimates of Prediction Error

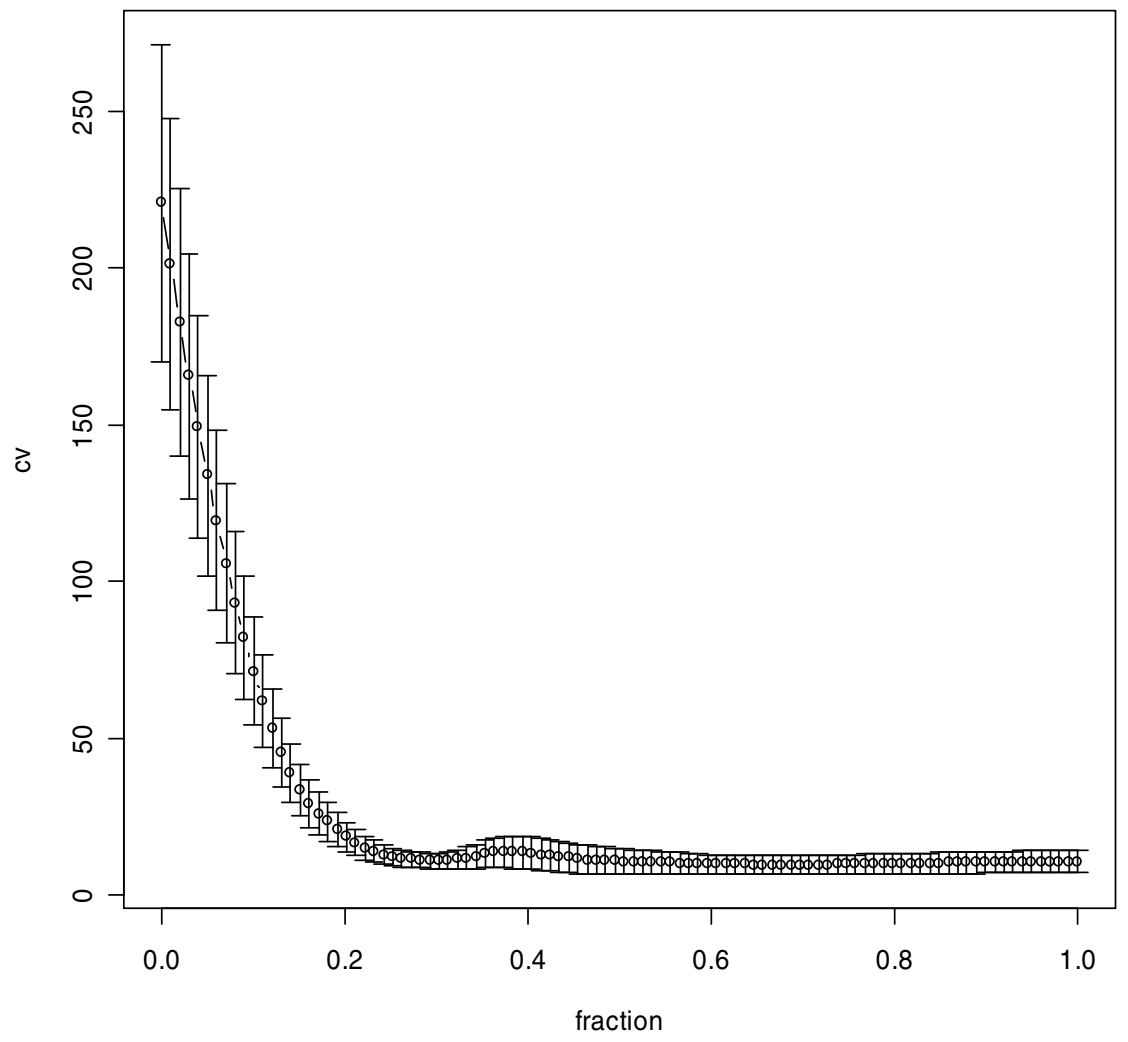


Figure S3: Cont'd

b) LAR, i) Plot of 10-fold Crossvalidation Values against Tuning Parameter LAR

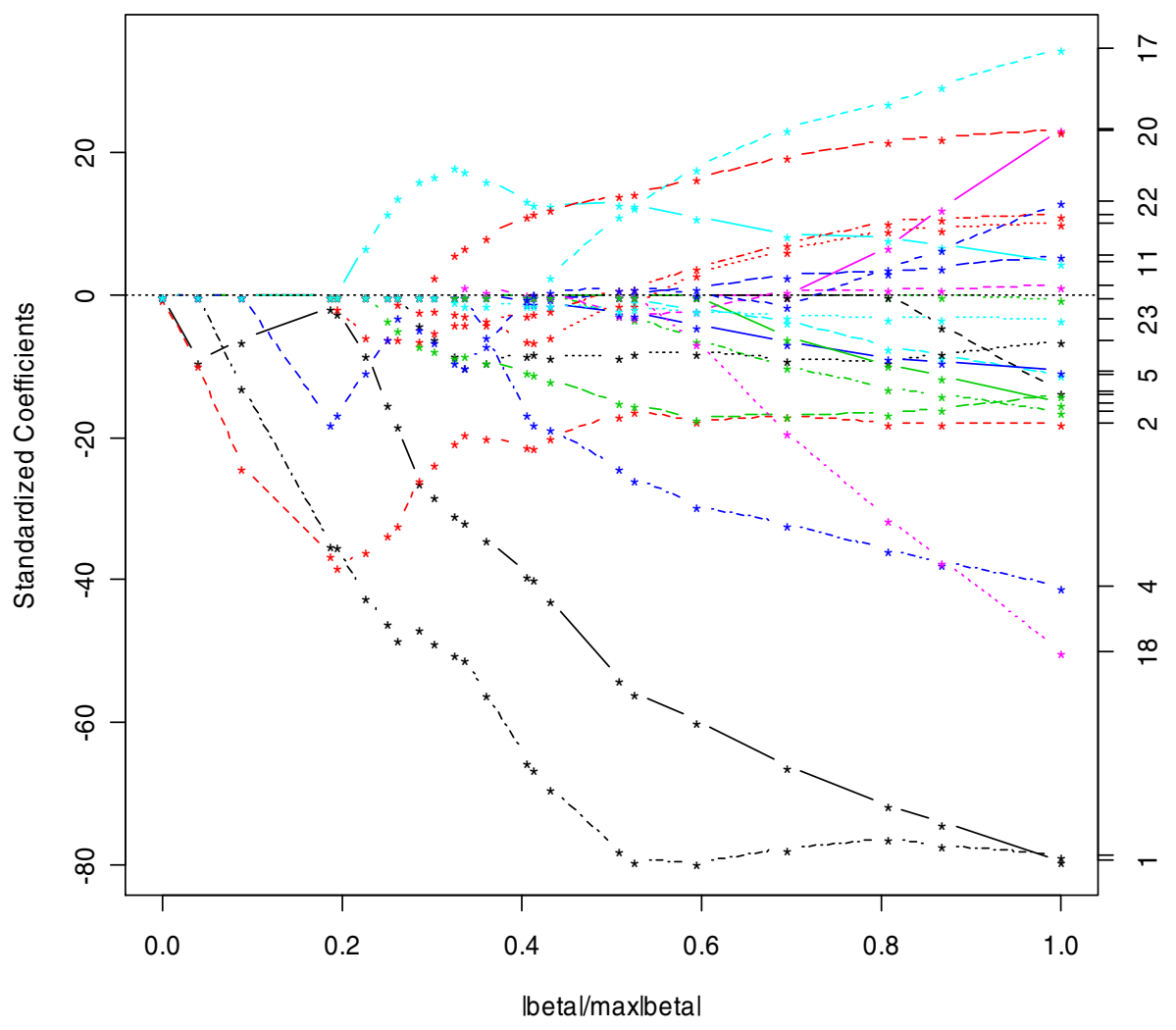

ii) Cross-validation Estimates of Prediction Error

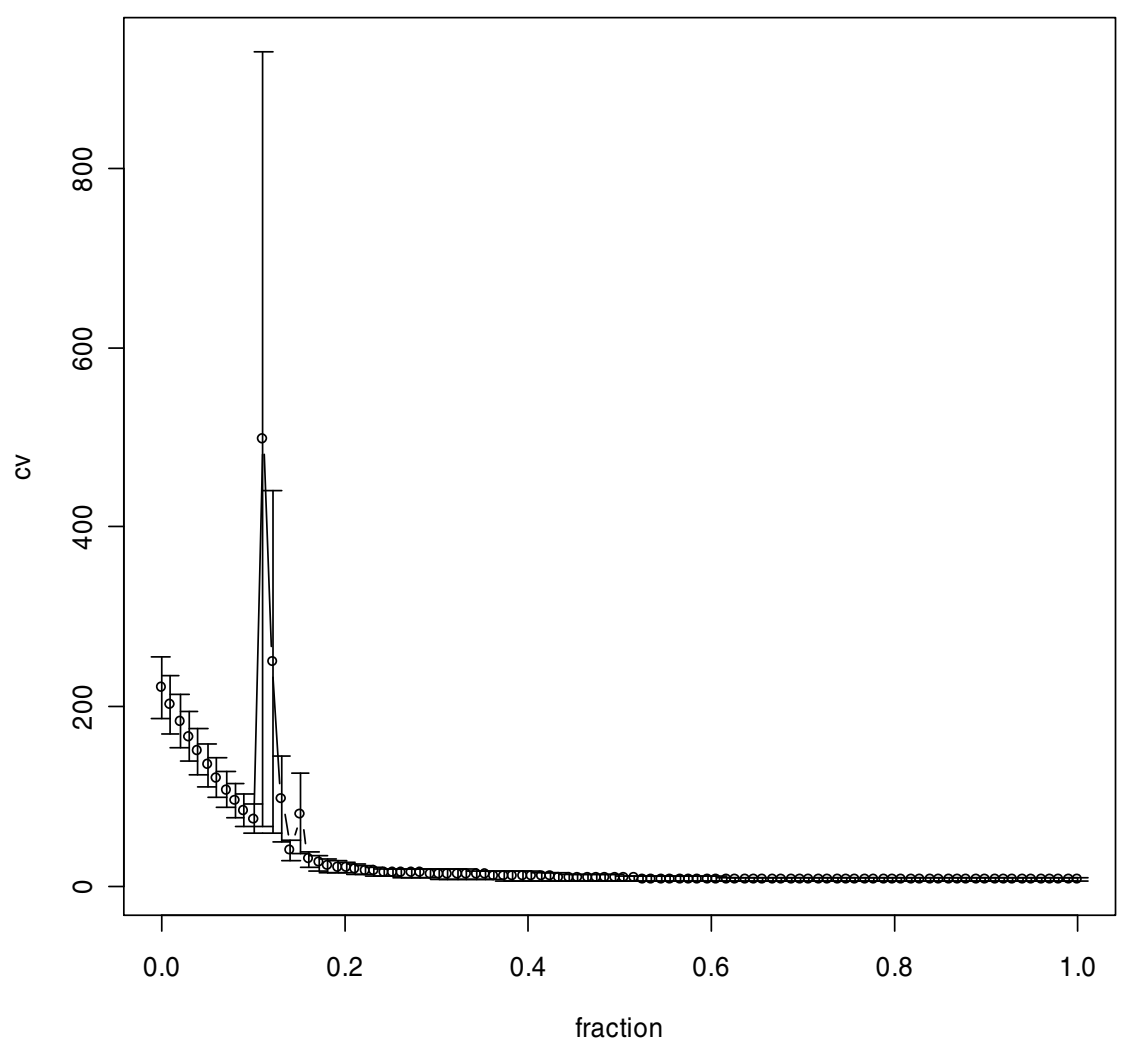


Figure S4: Diagnostic Plots for PCR Model.

i) Cross-validation Estimates of Prediction Error

PCR: 10 -fold CV

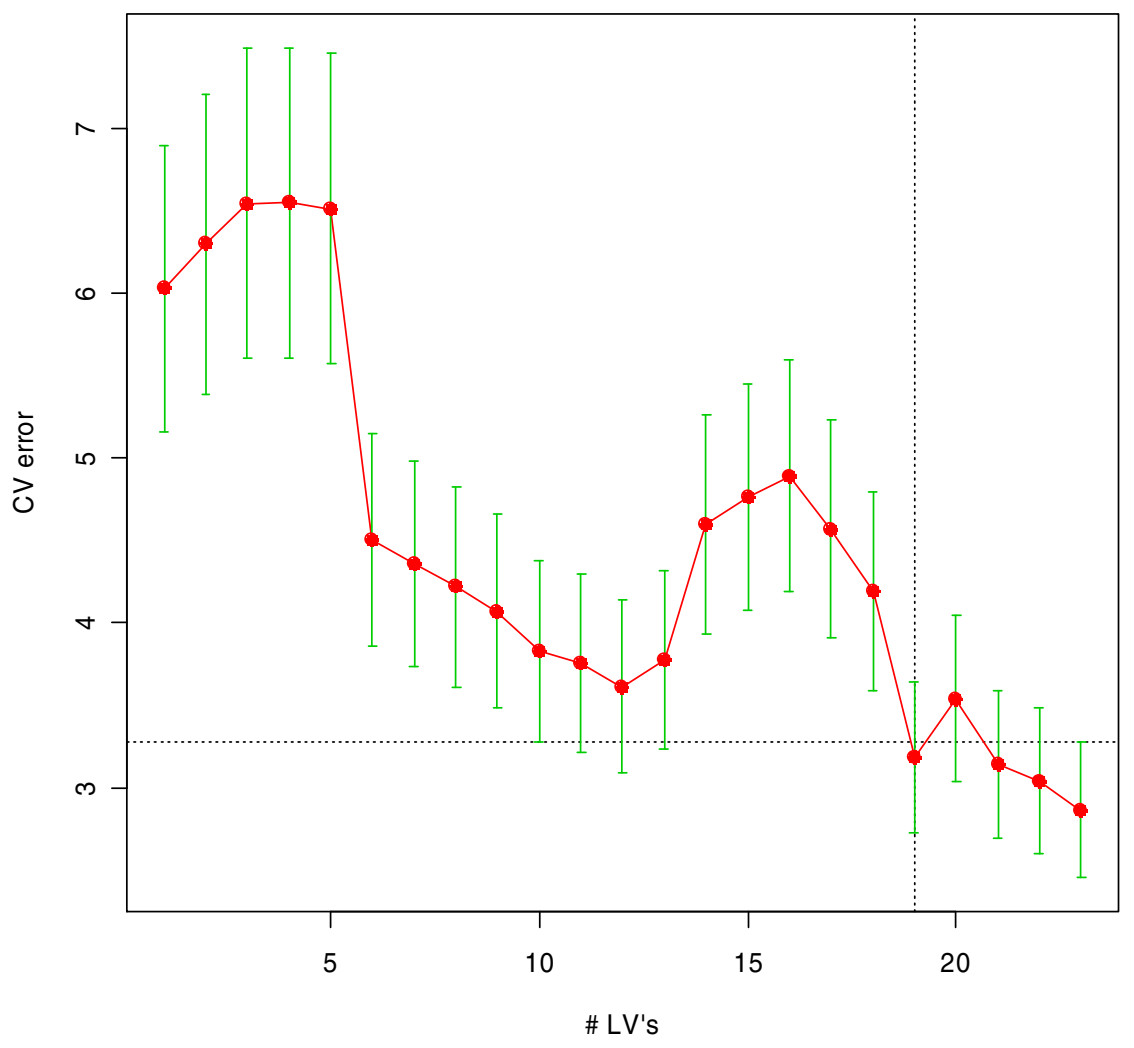

ii) Residuals vs. Fitted

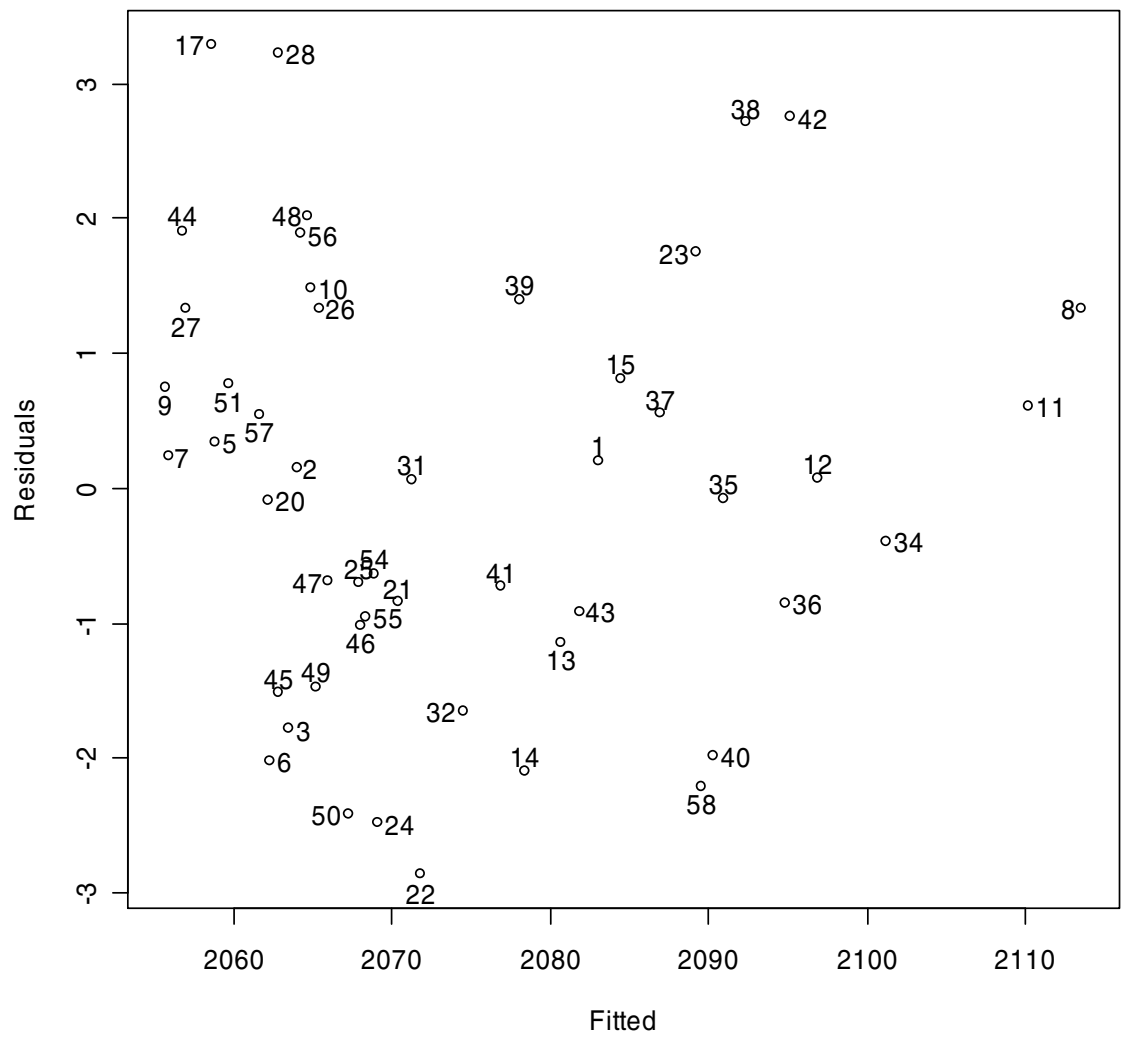


Figure S5: Diagnostic Plot for PLS Model

i) Cross-validation Estimates of Prediction Error

SIMPLS: 10-fold CV

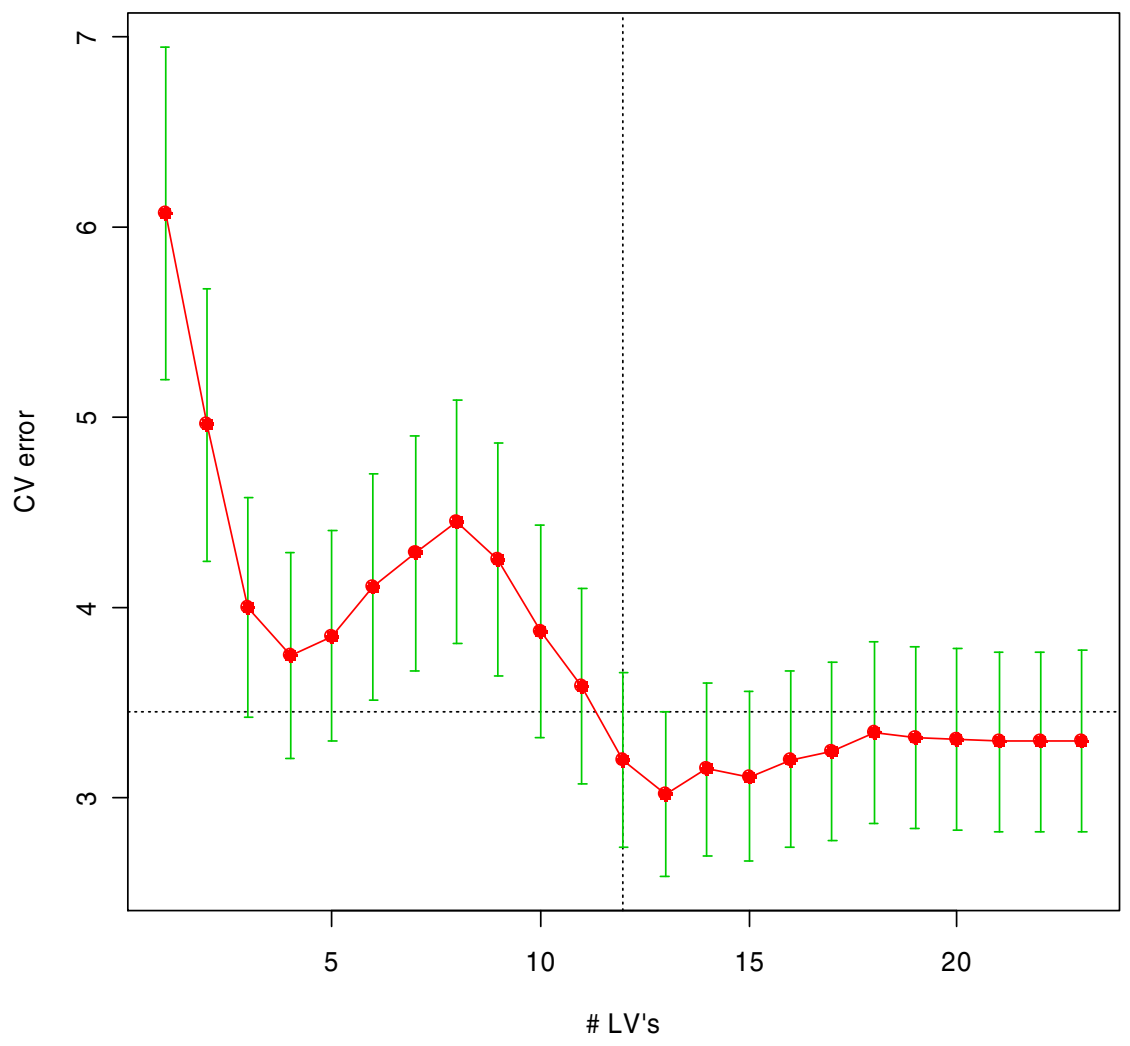

ii) Residuals vs. Fitted

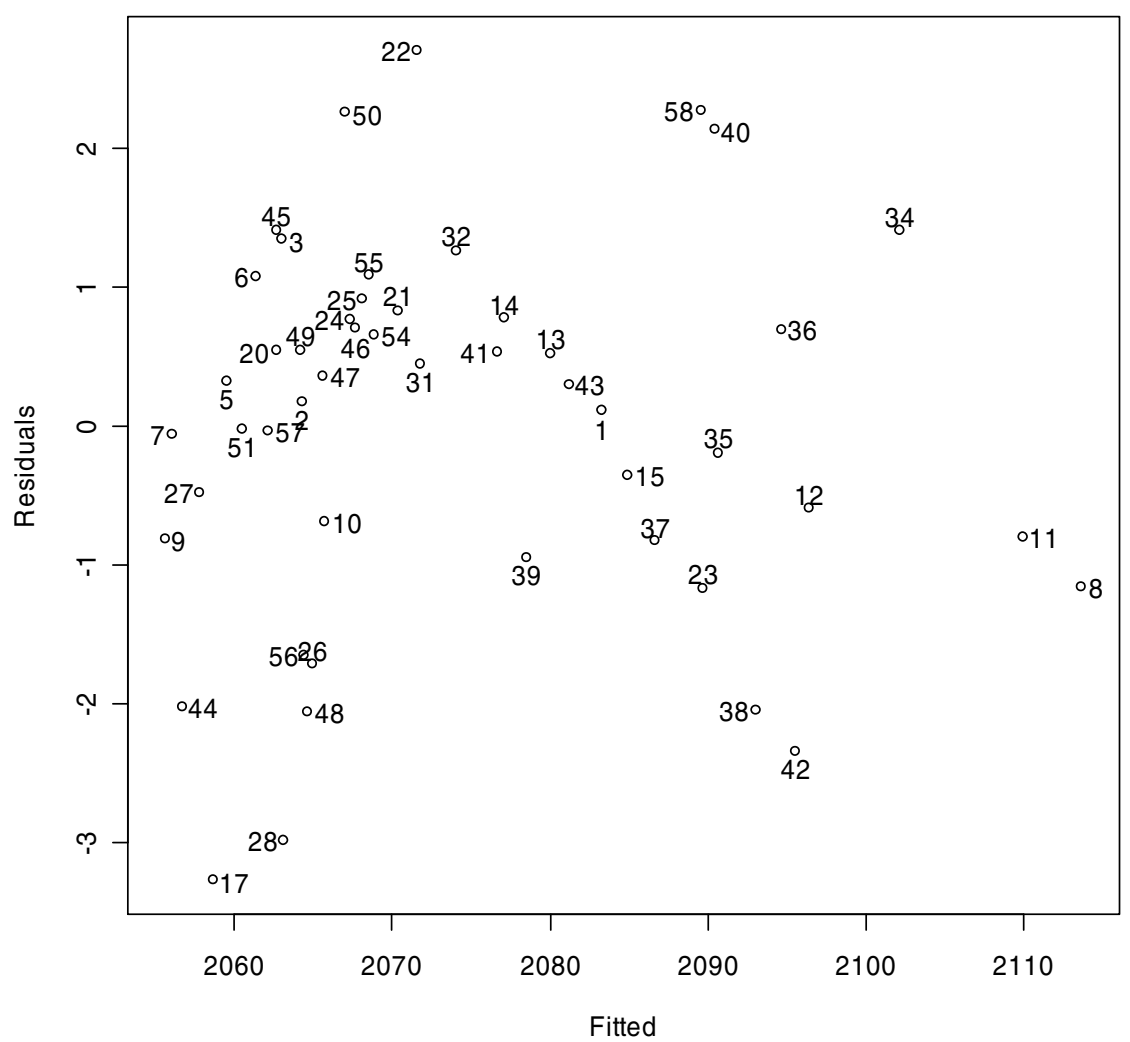

